\section{2}

\section{3}

\title{
A Study on the Effect of the Outer Ring of Annular Cooling Fan on the Aerodynamic Performance
}

\author{
Hengtao Shu ${ }^{1}$, Zhihong Yin ${ }^{1, *}$, Wenbin Shangguan ${ }^{1}$ and Waizuddin Ahmed ${ }^{2}$ \\ 1 School of Mechanical \& Automotive Engineering, South China University of Technology, Guangzhou, \\ People's Republic of China; 48973587@qq.com (H.S.); sgwb@scut.edu.cn(W.S.) \\ 2 CONCAVE Research Center, Mechanical \& Industrial Engineering, Concordia University, Montreal, \\ Canada; waiz.ahmed@concordia.ca; \\ * Correspondence: mezhyin@scut.edu.cn
}

\begin{abstract}
We studied the effect of the structure parameters of engine annular cooling fan with outer ring on the aerodynamic performance by means of experiments and model simulation in fluent ${ }^{\circledR}$. Firstly, based on the experiment, a computational model is developed to calculate and analyze the aerodynamic performance of the tested annular fan. The model is validated by comparing the test results with the calculated data. Besides, the aerodynamic performance differences between two types of fans (common fan without outer ring and annular fan with outer ring) are discussed. Based on the computational model, the relation between aerodynamic performance and the outer ring structure parameters are investigated. The results show that the relative parameter on the axial direction has great influence on the aerodynamic performance; while the effect of radial relative parameter is minor. In addition, the outer ring with arc chamfer structure in the downstream side can improve its static pressure efficiency effectively.
\end{abstract}

Keywords: annular cooling fan; outer ring; structure parameters; aerodynamic performance

\section{Introduction}

The performance of engine cooling fan includes aerodynamic performance and aerodynamic noise[1]. And the former is the primary consideration for designers, it includes the flow rate, the static pressure, the fan power and the static pressure efficiency. In the premise of a constant flow rate, the static pressure efficiency is expressed as:

$$
\eta=a \frac{q p_{s p}}{P}
$$

where $\eta$ is the static pressure efficiency, $q$ is the flow rate, $p_{s p}$ is the static pressure, $a$ is the coefficient, and $P$ is the power of the fan. Equation (1) shows that the static pressure efficiency takes both static pressure and fan power into consideration which makes it the most important aerodynamic performance index. Furthermore, the static pressure efficiency is usually the objective of structure optimization [2-4].

For annular cooling fan, an outer ring is added around the blade tip as shown in Figure. 1b, which is different from the common cooling fan shown in Figure. 1(a). 


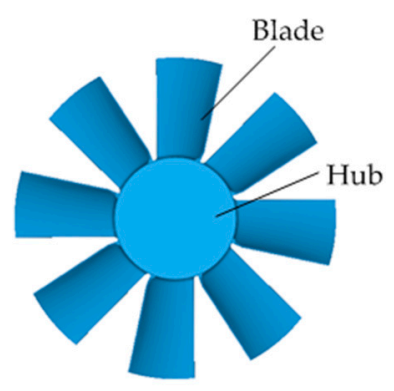

(a)

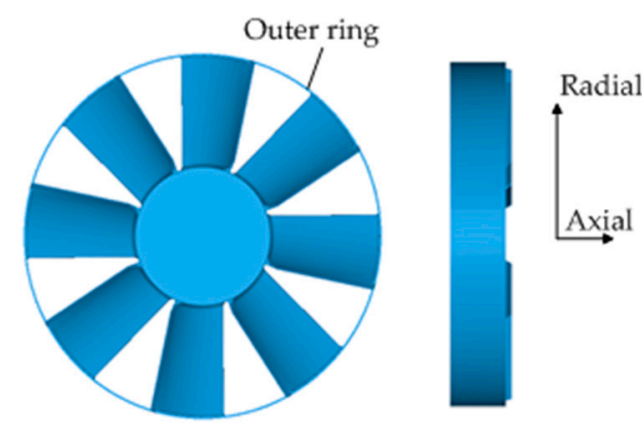

(b)

Figure. 1. Structure comparisons between (a) common fan without outer ring and (b) annular fan with outer ring

Annular fan is not widely applied in the engine cooling system due to the complex structure and high manufacturing cost. However, it has a salient advantage of having high static pressure and low energy consumption [5]. As the engine performance is strengthened, annular fan is receiving more and more interest. The usage rate of annular fan has been continually rising in heavy commercial vehicles, the flow friction and impulse impact losses are both reduced by the restriction of the outer ring. Therefore, the study of the engine cooling fan is mainly focused on annular fan.

The aerodynamic performances of an annular cooling fan are affected by many factors, which includes the whole diameter dimension, blade installing angle, blades number and hub ratio. Structure parameters of the outer ring will effect on aerodynamic performance in some way. However, the study on it is minor for annular fan.

We note that there are a number of international patents about annular fan in the field of vibrations and structural strength, but there are few papers to illustrate the design method of the outer ring in the field of aerodynamic performance optimization for annular cooling fan. Motivated at this problem, this paper aims to provide a systematic method to study the aerodynamic performance of an annular cooling fan. Specifically, our purpose is to investigate the effect of the outer ring and achieve some valuable conclusions for the outer ring design of the annular cooling fans. So this paper would facilitate the performance analyses and product development of annular cooling fan.

The main motivation of this paper is to present some proposals of outer ring design for annular cooling fan for the first time. All suggestions are obtained through a series of simulations and tests comparative analysis, and they are reliable and effective. The results will provide a certain engineering guiding in annular cooling fan design.

Generally, a common cooling fan is composed of a hub and blades. In the existing studies of structural optimization for cooling fan, researchers conducted a large number of studies on the hub ratio, and the aerodynamic performance is greatly improved [6-7]. However, many simulations in Fluent ${ }^{\circledR}$ have shown that the leakage locates on the tip of the blade is the main source of the aerodynamic performance loss for cooling fan [8]. It can be seen from Figure. 2 that an obvious backflow exists in the blade tip zone, which attributes to the blade tip leakage [9-10]. 


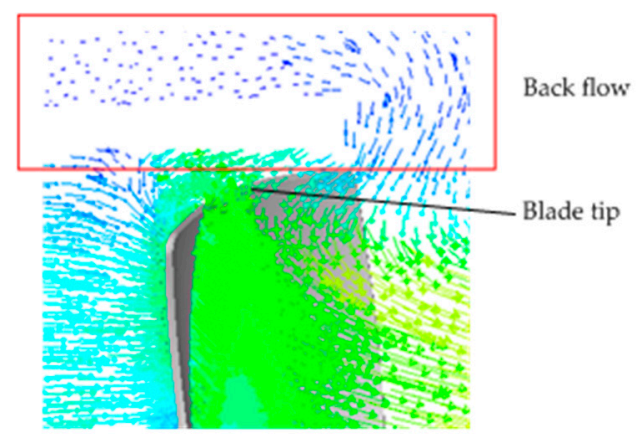

Figure. 2. Velocity contour profiles at one section of a cooling fan

For an axial flow machine, a clearance must be considered between the blade tip and the outer shell to ensure the relative motion for the two mechanical components. A pressure difference between the suction and the pressure surface is generated when the axial flow machine rotates for complex blade structure. In addition, the backflow is caused by the existence of blade tip clearance and the pressure difference [11]. The mixing flow on the blade surface is formed by the leakage vortex, and it brings axial aerodynamic performance degradation for the axial impeller machinery [12-15]. So, the tip leakage is one of the main sources of axial-flow energy loss. Furthermore, the tip leakage is the main source of aerodynamic noise [16-17]. It can be seen that the tip leakage has significant influence on the overall performance of the axial flow machinery.

The above discussions illustrate that the backflow caused by tip leakage is closely related to the reduction of the static pressure efficiency. The flow field of axial flow fan is very complicated. The backflow cannot be eliminated fundamentally, but can be relieved by structure optimization.

In Ref [18], in order to control the backflow and tip leakage, a rapid air injection device was assembled in the inlet section of a compressor. A drawback of this design of adding auxiliary device on the blades periphery is that it brings a higher production cost. However, this design method of adding an auxiliary device to improve the aerodynamic performance provided an optimization idea to restrain tip leakage for an axial cooling fan. In addition, it gave a push to the birth of the annular cooling fan.

The outer ring structure was evolved from winglet [19]. In the earliest studies, a winglet was added at the intermediate position of compressor blades to reduce the noise level [20-22]. By adding the winglet on the blades, the natural frequency was changed too [20-21]. Furthermore, the simulation results $[5,23]$ indicate that the aerodynamic performance was improved obviously.

Compared with the common axial fan, the annular fan has stronger pressure lift capacity, and it is initially used as a lift fan in aircraft. Many studies have been done on how to improve the aerodynamic performance of the fan mentioned above. For example, the inlet lip radius and diffuser angle were optimized multiple times to improve the lift efficiency of the fan [24].

The above literatures are all about compressors, and the engine cooling fans belong to the axial mechanical as well as compressors. The aerodynamic performance was improved by optimizing the structure of auxiliary equipment for annular fan $[17,25]$, but there were few studies on optimizing the structure of the fan itself. Based on the optimization methods in the Refs [18, 25], an instance annular automobile engine cooling fan is studied in this paper. The emphasis is put on studying the influence of the outer ring structure parameters on the aerodynamic performance for the annular cooling fan.

The organization of this paper is as follows: in section 2, based on the experiment condition of the annular fan, a computational model is developed in Fluent ${ }^{\boxplus}$. The data post-processing is used to analyze the aerodynamic performance of the target fan, including the flow rate, static pressure, fan power and static pressure efficiency. The model is validated by comparing the experimental data with the modeling results. Based on the computational model, some qualitative relationships are 
102

103

104

105

106

107

108

109

110

111

112

113

114

115

116

117

investigated between aerodynamic performance and the outer ring structure parameters. In section 3 , three kinds of structure parameters of the outer ring are presented. In section 4, all annular cooling fans that change the corresponding structure parameters are simulated and discussed.. The design method of the outer ring is presented and validated in this section. Conclusions are provided in section 5 .

\section{Methods}

During the structure optimization process of fluid machinery, the application of Fluent ${ }^{\circledR}$ is able to improve the efficiency in investigating a new cooling fan. The simulation model adopt in Fluent ${ }^{\circledR}$ is the key process for the cooling fan structure optimization. To guarantee a good accuracy of the simulation model, the simulation results results should be benchmarked based on the test results.

\subsection{Experiment Setup and Evaluation Indexes}

As aforementioned, there are three aerodynamic performance evaluation indexes for cooling fan, i. e. static pressure, fan power and static pressure efficiency.

Based on Ref. [1], an aerodynamic performance test bench for the cooling fan is set up, and the schematic diagram is shown in Figure. 3. The test bench mainly consists of three parts: the air-flow pipe, the mechanical transmission and the test control board.

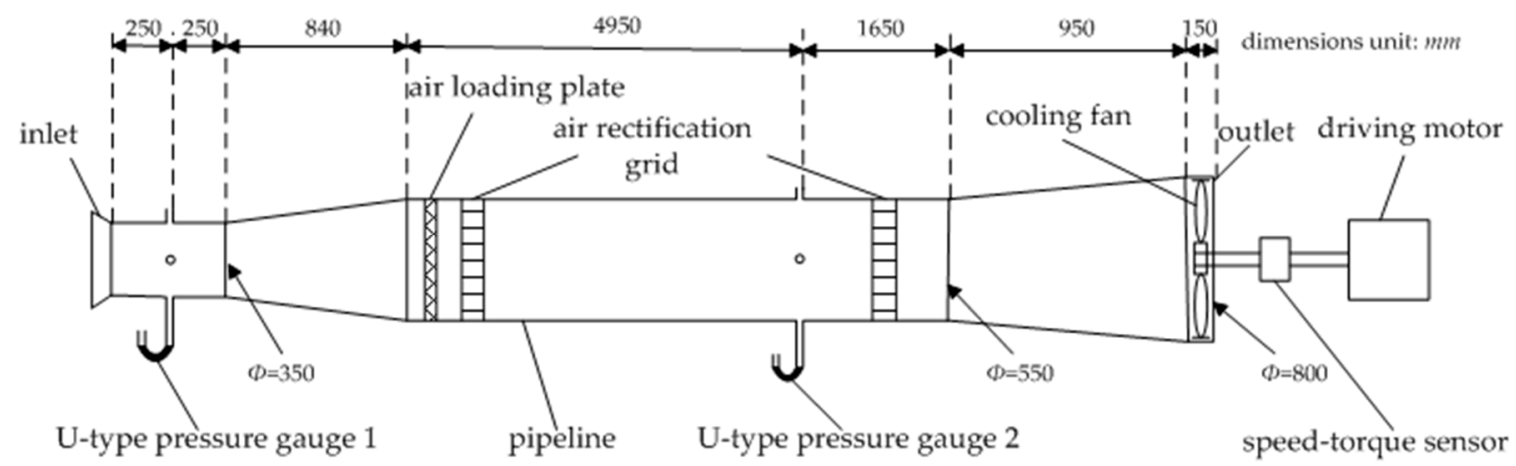

Figure. 3. The schematic of fan aerodynamic performance test bench

118

119

120

121

122

123

124

125

126

The pipeline has both inlet and outlet space. Both the upstream side of the inlet space and the downstream side of the outlet space are connected to the air, which can be treated as 0 Pa (relative value) space. The mechanical transmission section locates at the downstream side of the outlet, and the hub of the cooling fan is connected to the driving motor through the speed-torque sensor. The data of rotating speed $(n)$ and torque $(T)$ of the cooling fan can be measured instantly by the sensor. The flow rate $(q)$ in the pipeline can be set optionally by changing different air loading plates, which have different flow holes sizes within them. Two U-type pressure gauges are installed in different locations along the flow direction. The static pressure at the inlet $\left(p_{s i}\right)$ and monitoring section $\left(p_{s o}\right)$ can be both measured by the tow gauges. The photograph of the test bench is shown in Figure. 4 . 


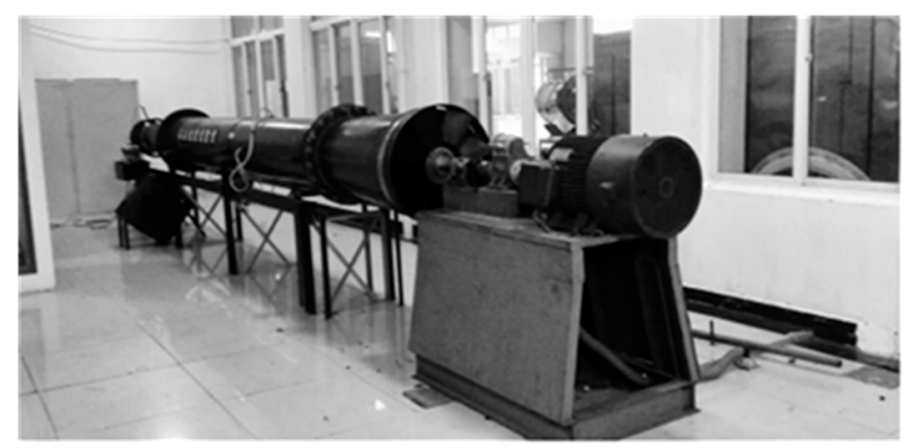

Figure.4. The photograph of fan aerodynamic performance test bench

Based on the working principle of the test bench, the flow rate mentioned in this paper means the volume of external air-flow that flows through the cooling fan per unit of time. it can be calculated by the static pressure measured by the U-type pressure gauges 1 , which can be expressed as:

$$
q=\alpha_{\varepsilon} \frac{\pi d^{2}}{4} \sqrt{\frac{2\left|p_{s i}\right|}{\rho}}
$$

where $\alpha_{\varepsilon}$ is the air flow coefficient number, and it approximately equals 0.96 in this paper [1]; $d$ is the diameter of the duct section where U-type pressure gauge (pitot tube) locates at the inlet side; $p_{s i}$ is the static pressure at the same section, which is measured by the gauge 1 as shown in Figure. 3; and $\rho$ is the air density.

The total pressure $\left(p_{t p}\right)$ of the air at a certain section of the bench is composed of static pressure $\left(p_{s p}\right)$ and dynamic pressure $\left(p_{d p}\right)$. The total pressure $p_{t p}$ can be measured by the pitot tube, so do the $p_{s p}$, which is generated from pressure perpendicular to the duct wall. Based on the above description, $p_{d p}$ can be calculated which is the difference between $p_{t p}$ and $p_{s p}$. The total pressure of the cooling fan is defined by the total pressure difference between the section of the outlet and inlet, which can be expressed as:

$$
p_{t p}=\left(p_{s p 2}+p_{d p 2}\right)-\left(p_{s p 1}+p_{d p 1}\right)
$$

The static pressure of cooling fan is defined by the difference between the total pressure and the dynamic pressure at the outlet section, which can be expressed as:

$$
p_{s p}=p_{t p}-p_{d p 2}=p_{s p 2}-\left(p_{s p 1}+p_{d p 1}\right)
$$

Because of the complex flow-field of bench ducts, the pressure loss $(\Delta p)$ is composed of frictional resistance loss $\left(\Delta p_{f}\right)$ and local pressure loss $\left(\Delta p_{l}\right)$, based on the test experience [1], the $\Delta p$ can be expressed as:

$$
\Delta p=\Delta p_{f}+\Delta p_{l}=\frac{p_{d p 2}}{10}
$$

Based on (3)-(5), the static pressure of the fan can be expressed as:

$$
p_{s p}=p_{s p 2}-\left(p_{s p 1}+p_{d p 1}-\Delta p\right)=-p_{s p 1}-\frac{1}{2} \rho\left(\frac{q}{A_{1}}\right)^{2}+\frac{p_{d p 2}}{10}
$$


By the transfer pass of energy in the air duct, the fan power $(P)$ consists of fan power loss $\left(P_{p}\right)$ and effective fan power $\left(P_{e s p}\right)$. The fan power of the fan is equal to the energy transmitted from the driving motor, which can be expressed as:

$$
P=\frac{T n}{9550}
$$

Here, $T$ is the output torque of the driving motor, $n$ is the rotating speed of the cooling fan. These values are all measured by the speed-torque sensor as shown in Figure. 3.

The effective fan power is equal to the energy absorbed by air when it flows through the rotating fan during per unit time, which can be expressed as:

$$
P_{e s p}=\frac{q p_{s p}}{1000}
$$

\subsection{Simulation Model}

As shown in Figure. 1, the 3D cooling fan model is established, and the fan-hub is simplified Based on the construction of the test bench, the simulation model is developed by Fluent ${ }^{\circledR}$, as shown in Figure. 5. During the simulation, the input flow rate can be set arbitrarily based on the actual test condition. Besides, the static pressure can be obtained directly by the monitoring section data. In addition, a rotating fluid zone with suitable size is developed. The outlet downstream area is directly connected to the air, so a cylindrical duct with the diameter and length of $4 \mathrm{~m}$ is developed. Some transition regions are established to smooth every flow zone along the direction from the inlet to the outlet.

In order to improve the computational accuracy and reduce the grid number, the tetrahedral grids are employed in the rotating fluid and transition zones, and the hexahedral grids are employed in the inlet and outlet flow duct. In addition, these two different types of grids can be used synthetically to meet actual calculation requirements.

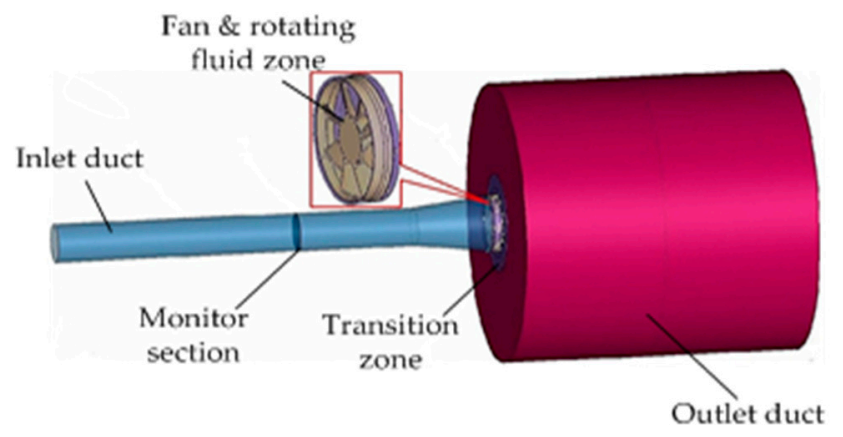

Figure. 5. The computational model

\subsubsection{Grid Independent Validation}

Under a steady state numerical calculation, the grid independence is necessary to be verified. Four grid plans are presented in Table 1.

Table 1. Results of grid independent validation

$\begin{array}{llllll}\text { Plan serial } & \text { Grid } & \text { Grid } & \text { Volume flow rate } & \text { Rotating speed } & \text { Static pressure }\end{array}$




\begin{tabular}{cccccc}
\hline number & size & number & $\left(\mathbf{m}^{3} / \mathbf{s}\right)$ & $(\mathbf{r p m})$ & $(\mathbf{P a})$ \\
\hline 1 & 10 & 924,573 & 2.81 & 1500 & 654 \\
2 & 8 & $1,276,384$ & 2.81 & 1500 & 699 \\
3 & 6 & $1,713,261$ & 2.81 & 1500 & 685 \\
4 & 4 & $2,238,734$ & 2.81 & 1500 & 689 \\
\hline
\end{tabular}

Note: The rotating fluid zone where the cooling fan locates is the most critical zone for the computational model as shown in Figure. 5. Therefore, the grid size mentioned in the table denotes the wall size of the rotating fluid zone; and the grid number denotes the number of grid in the same zones.

In this part, the static pressure is used to be the evaluation index to determine which plan is finally to be adopted. The calculation is implemented with the flow rate is $2.81 \mathrm{~m}^{3} / \mathrm{s}$. The static pressure value differences of the four plans are less than $2 \%$. In order to reduce the computational burden, the Plan 2 with the fewest grids is considered in this paper.

\subsubsection{Simulation Algorithm Selection}

The multi-reference frame method (MRF) is a robust algorithm, which is computationally efficient with acceptable accuracy. Therefore, the MRF method is adopted to calculate the aerodynamic performance of the cooling fan in this paper. The flow rate loading panel is used to change the flow rate in the pipeline. Therefore, the inlet section is set as the mass-flow inlet boundary, where the mass flow can be changed arbitrarily to meet the need of the simulation. The outlet section is set as the pressure outlet boundary and the static pressure monitoring section is set as the interior boundary.

Furthermore, the fan blade surface is set as the rotating wall boundary. The remaining surfaces are set as stationary wall boundaries.

The airflow condition in the test pipeline belongs to low-speed flow (the Mach number is less than 0.3). Therefore, the air in the flow field is considered as in-compressible medium. The SIMPLE algorithm is used in the pressure-velocity coupling equations and the RNG k- $\varepsilon$ model is used in this model. Besides, the wall function method is used to implement numerical iterative in near wall area. The separation formula solution is used for the solver setup. When the flow rate difference between the inlet flow and the outlet flow is less than $0.5 \%$, the calculation result is considered to be convergent.

\subsection{Model Verification}

In this section, a common engine cooling fan without outer ring is selected as an example for demonstrations. It has a diameter of $760 \mathrm{~mm}$ and 8 blades which are equidistantly distributed (as shown in Figure. 1a). Based on the actual condition, the fan rotating speed is set to $1500 \mathrm{r} / \mathrm{min}$. The cooling fan rarely works within a low flow rate region. Therefore, the middle and high flow rate regions in steady operating condition are considered in this paper. Comparisons between the simulation and experiment data are shown in Figure. 6. 


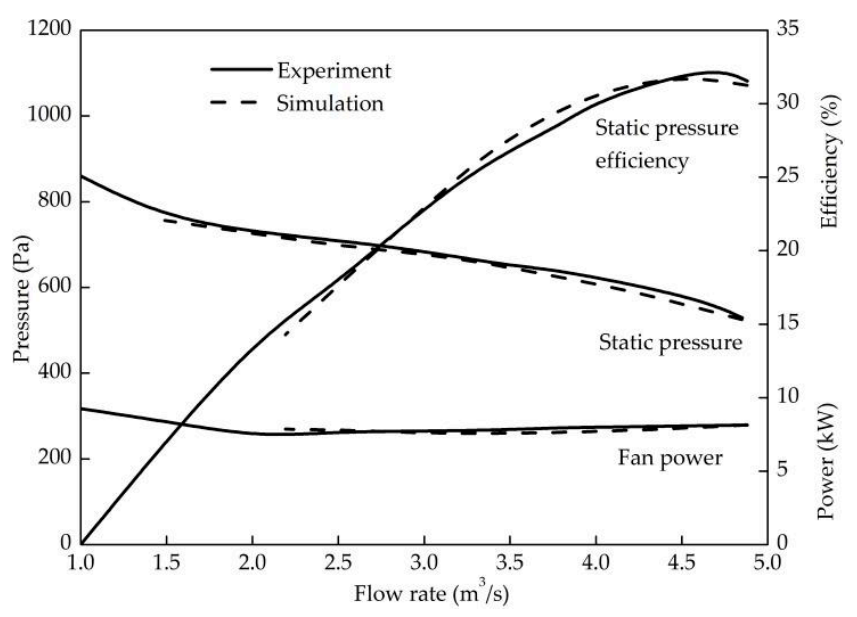

Figure. 6. Aerodynamic performance comparisons between experiment and simulation results

As shown in Figure. 6, the static pressure of the cooling fan decreases with increases of flow rate. The fan power changes slightly with increases of flow rate. Besides, the static pressure efficiency firstly increases and then decreases with the increases of flow rate. In addition, it has the same tendency as the static pressure efficiency. Furthermore, the difference becomes larger as the flow increases. It can be found from Figure. 6 that the errors are all less than $10 \%$ between the simulation and experiment results. Therefore, the simulation model can be used to predict the aerodynamic performance of the cooling fan.

\section{Structure and Parameters of Outer Ring}

The external physical characteristics of annular fan with outer ring are shown in Figure. 7. Three dimension structure parameters about the outer ring will be described in this section: the axial projection width $(W)$, the diameter $(D)$ and its shape.

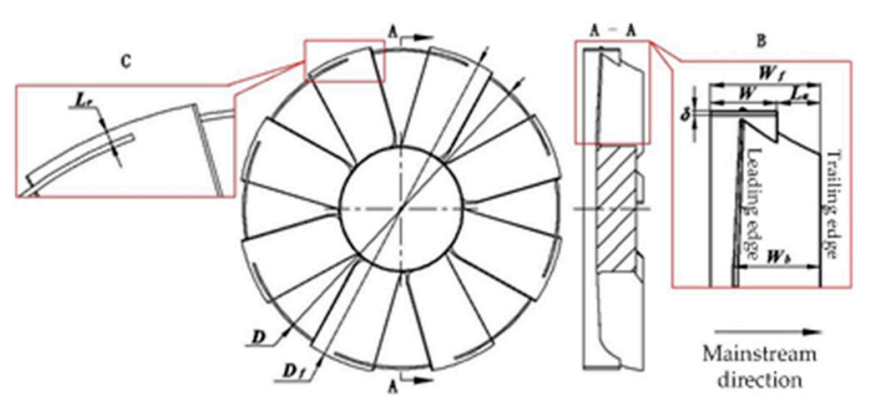

Figure. 7. Structure parameters of annular fan with outer ring

\subsection{Axial Structure Parameter}

First of all, all axial lengths in this paper are projected along the airflow direction. $i$ is a custom axial structure parameter being discussed in this paper that is termed as "aperture opening ratio", and it can be expressed as:

$$
i=\frac{W_{f}-W}{W_{b}} \times 100 \%
$$

In Figure. $7, W_{f}$ is the total axial length of the annular cooling fan, $W$ is the width of the outer ring, and $W_{b}$ is the axial length of the fan blade. 
Furthermore, based on the parts assembly requirements, a certain distance must be kept between the front-edge-wall of the outer ring and the blade trailing edge. Therefore, $i$ is always lager than zero.

\subsection{Radial Structure Parameter}

As aforementioned, the winglet that is added on fan blades can change the inherent frequency of the fan by changing its radial installation position. The outer ring is evolved from it to improve the aerodynamic performance in this paper. Therefore, $L r$ is another custom radial structure parameter termed as "overhang length" in this paper, which is expressed as:

$$
L_{r}=\frac{D_{f}-D}{2},
$$

Based on Figure. 7, $D_{f}$ is the diameter of the fan blade, and $D$ is the external diameter of the outer ring. In addition that the outer ring is connected to the fan blade, and $L r$ is nonnegative.

\subsection{Shape}

The flow state can be changed or even improved by setting flared structure for the duct flow field. Based on the flow direction, the flared structure is divided into forward and reverse directions. As shown in Figure. 8, based on the flat ring structure (shape 3), arc chamfer is performed on each side of the outer ring respectively to study the influence of different shapes of the outer ring on the aerodynamic performance.

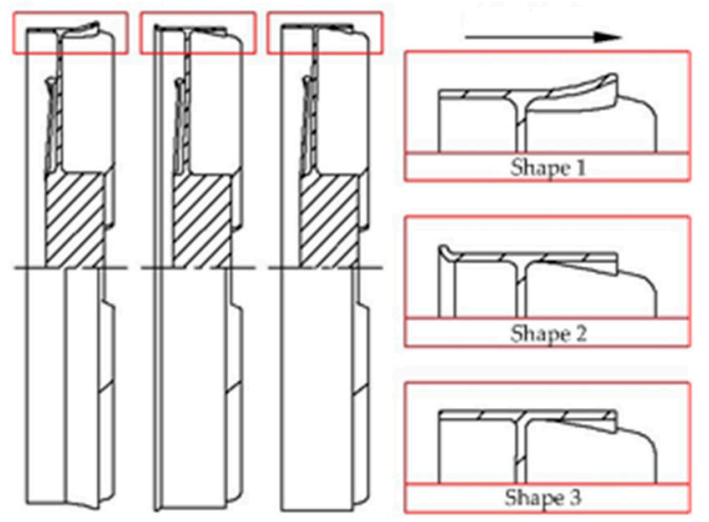

Figure. 8. Outer ring with 3 different shapes

All the structural parameters of the outer ring have been discussed. Furthermore, two fans are shown in Figure. 1 are taken as examples to be calculated to achieve the following goal: improving the aerodynamic performance by the design of adding the outer ring to the common fan.

\subsection{Aerodynamic Performance Comparison for 2 Kinds of cooling fan}

Both common and annular cooling fans are calculated by the model described in section 2, and the calculation results are shown in Figure. 9.

Based on Figure. 9, it can be found that the aerodynamic performance of the annular fan with outer ring is obviously better than that of the common one without outer ring except for the fan power. The fan power loss of the annular cooling fan increases due to the added weight of the outer ring. However, the fan power changes so slight that the change can be neglected The air will gather around the fan for the outer ring when it flows through the pipeline (as shown in Figure. 10). Hence, it can reduce the energy consumption along the air flow path. 


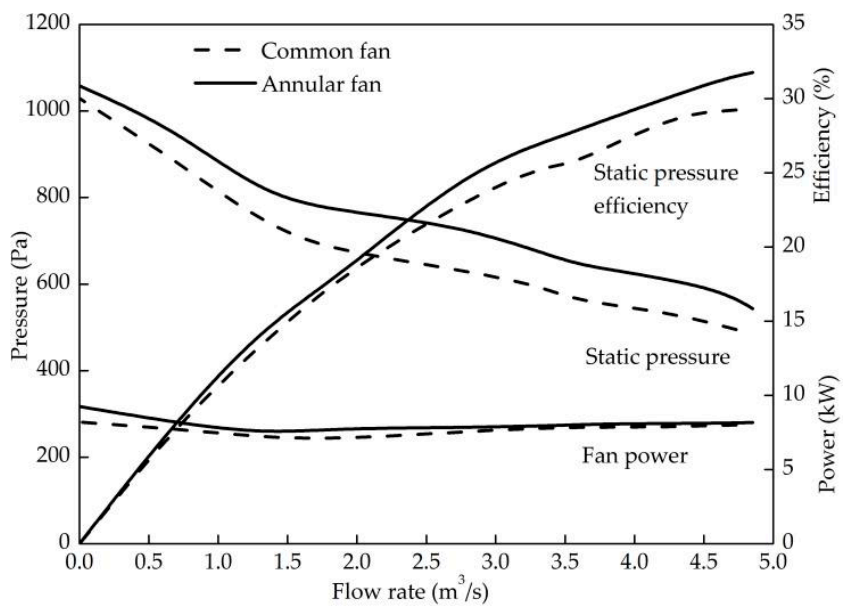

Figure. 9. Aerodynamic performance comparisons for common fan without outer ring and annular fan with outer ring

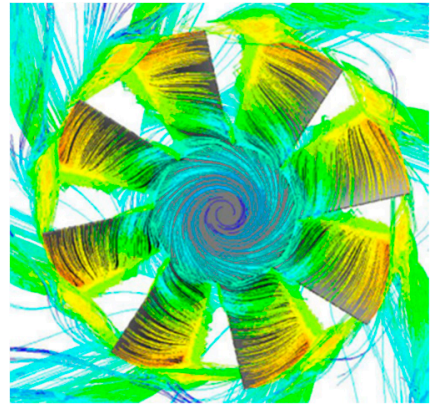

(a)

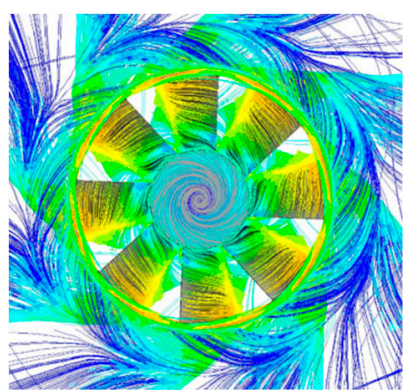

(b)

Figure. 10. Velocity contour profiles comparisons for (a) common fan without outer ring and (b) annular fan with outer ring (radial direction)

The outer ring improves the aerodynamic performance, and the change of the air flow contour profiles in the backflow zone will be observed along the axial direction. The backflow zones are marked by the red box as shown in Figure. 11 .

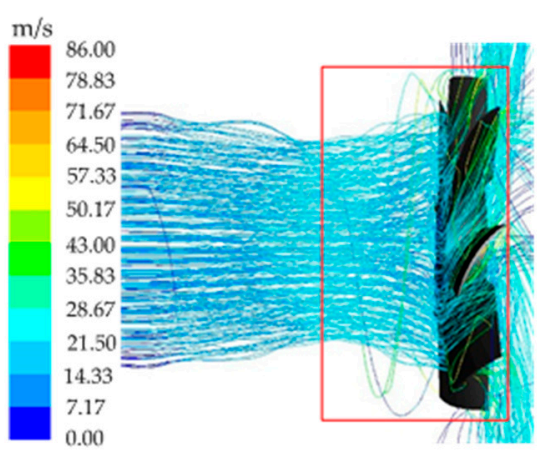

(a)

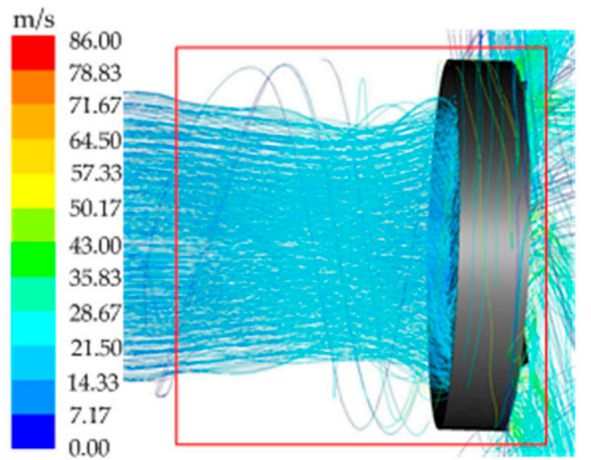

(b)

Figure. 11. Velocity contour profiles comparisons for (a) common fan without outer ring and (b) annular fan with outer ring (axial direction) 
Based on the distribution of the velocity contour profiles, there exist backflow area for both without and with outer ring cooling fans. Furthermore, it is larger for the fan with outer ring. However, the velocity value of backflow is a larger for the fan without outer ring. As a result, the structure of outer ring can guide the backflow forcibly and reduce the back flow degree. The static efficiency of an annular fan is improved spontaneously.

\section{Discussion}

In above section, the structure parameters of the outer ring have been detailed defined. In this section, the influence of the structure parameters on the aerodynamic performance of the outer ring are analyzed one by one. Furthermore, some design laws of the outer ring with certain guide significance can be found in the last part of this section.

\subsection{Effects of the Axial Structure Parameters}

Based on the discussions in above sections, the aperture opening ratio $i$ is defined as the axial structure parameters of the outer ring to be studied. In this section. Six annular fans with arbitrary aperture opening ratios are calculated, and results are shown in Figure. 12.

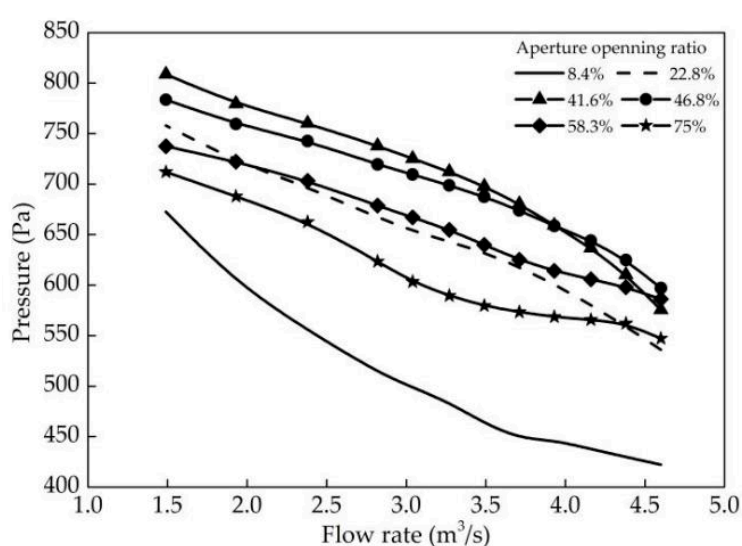

(a)

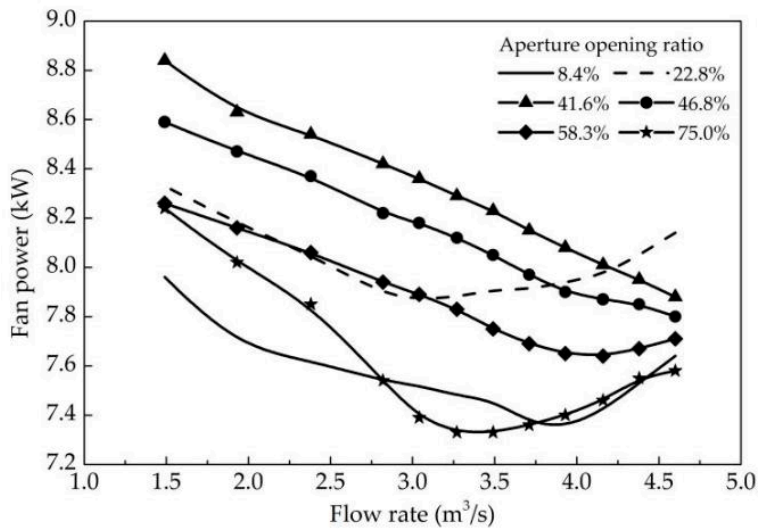

(b)

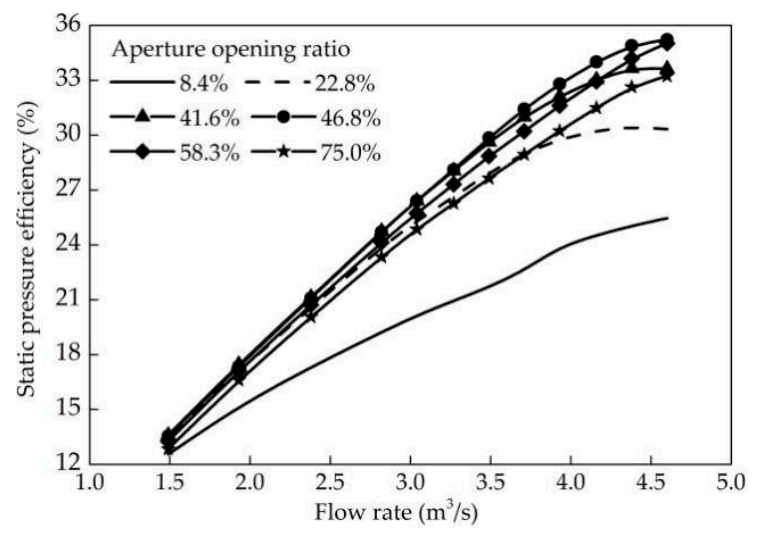

(c)

Figure. 12. Aerodynamic performance curves of (a) static pressure (b) fan power and (c) static pressure efficiency of annular fan with outer ring with different aperture opening ratios

It can be found from Figure. 12 that annular cooling fans with different $i$ have different aerodynamic performances. The value of static pressure efficiency is the lowest when $i=8.4 \%$. With the increases of $i$, the static pressure efficiency also increases. When the opening rate is $46.8 \%$, the 
270

efficiency reaches the top, after which, it decreases with the increases of $i$. This phenomenon will provide a good guidance for the annular cooling fan design.

As discussed in section 1, the back flow can disturb the air flowing direction and result in efficiency decrease of cooling fan. In Figure. 13, the back-flow area of three annular cooling fans with different aperture opening ratio outer rings are compared. The backflow area value is the smallest when $i=46.8 \%$, and it has the optimal aerodynamic performance, which is in accords with the change tendency as shown in Figure. 12.
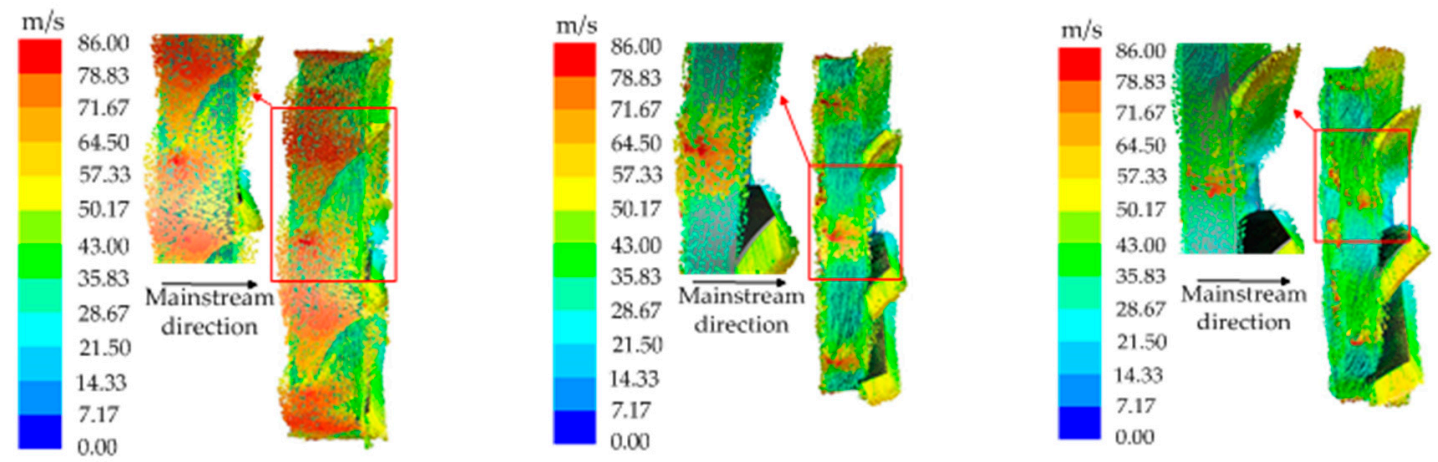

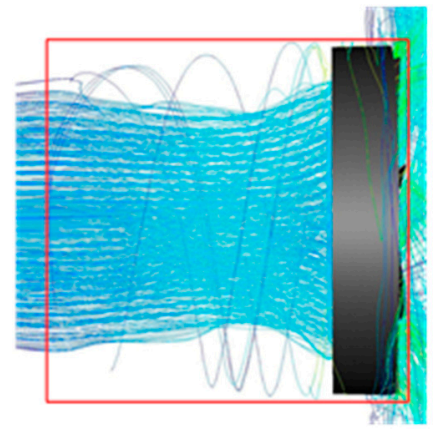

(a)

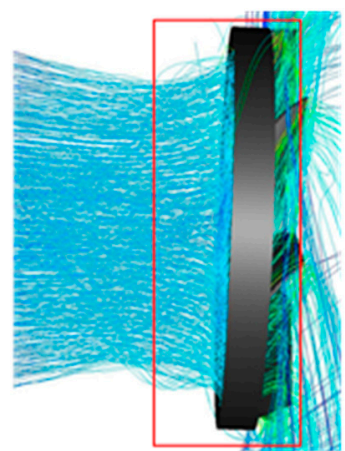

(b)

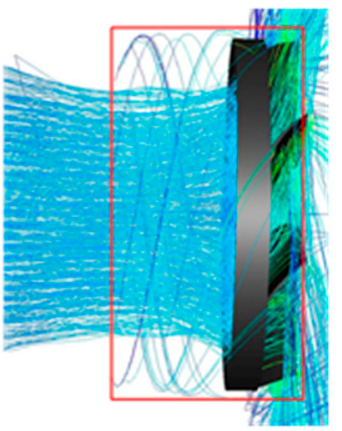

(c)

Figure. 13. Velocity contour profiles comparisons for annular fans with different aperture opening ratio outer

$$
\text { rings: (a) } i=8.4 \% \text { (b) } i=4.8 \% \text { and (c) } i=75.0 \%
$$

It can be found that the aperture opening ratio of the outer ring has a great effect on the aerodynamic performance. However, they are not simply positively or negatively related. Combing the primary evaluation index of static pressure efficiency, we infer that $41.6 \%$ to $46.8 \%$ is the optimal aperture opening ratio range for the annular cooling fan.

\subsection{Effects of the Radial Structure Parameters}

As mentioned in section $3, L r$ is defined as the radial relative parameter of the outer ring. Based on the above discussions, an annular fan with the aperture opening ratio of $46.8 \%$ outer ring is adopted for the investigation in this section. Four simulations of annular fans with different radial blade extensions $(L r=0,10,20,30 \mathrm{~mm})$ are carried out. The results are shown in Figure. 14. 


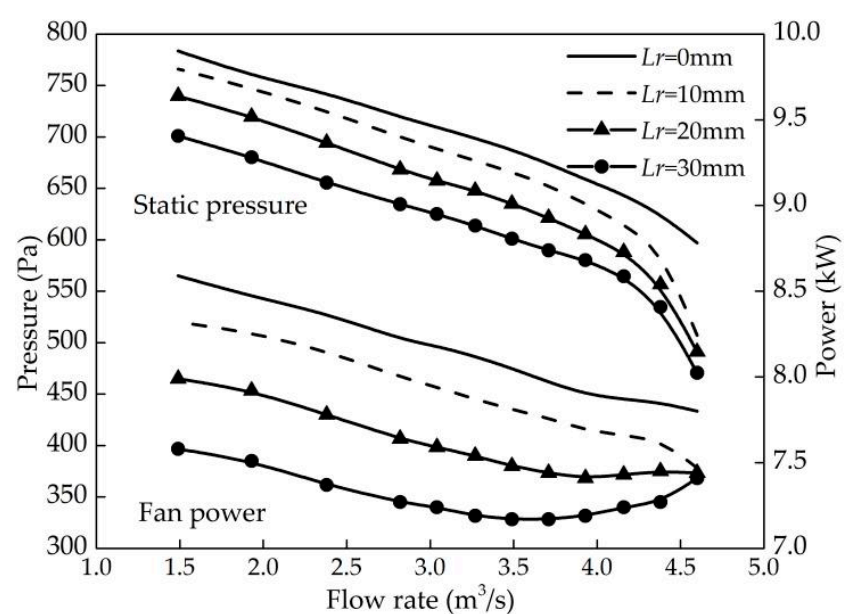

(a)

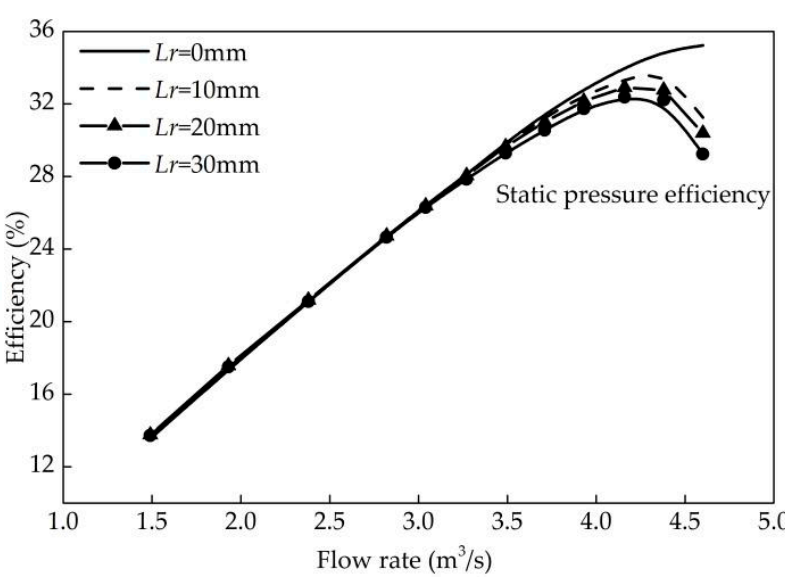

(b)

Figure. 14. Aerodynamic performances comparisons for annular fans with with 4 different radial blade extension outer rings: (a) static pressure \& Fan power, (b) static pressure efficiency

Based on Figure. 14, we can find that: 1) both the static pressure and fan power decrease with the increase of $L r$ under the same flow rate, 2) the static pressure efficiency in the high flow rate region also decreases with the increase of $L r$.However, in the middle-low flow rate region, the static pressure efficiency does not change obviously. Therefore, it comes to a conclusion that the radial blade extension has a slight effect on the static pressure efficiency. The same flow field analysis method is used in this section, as shown in Figure. 15, to illustrate the effect of this parameter.
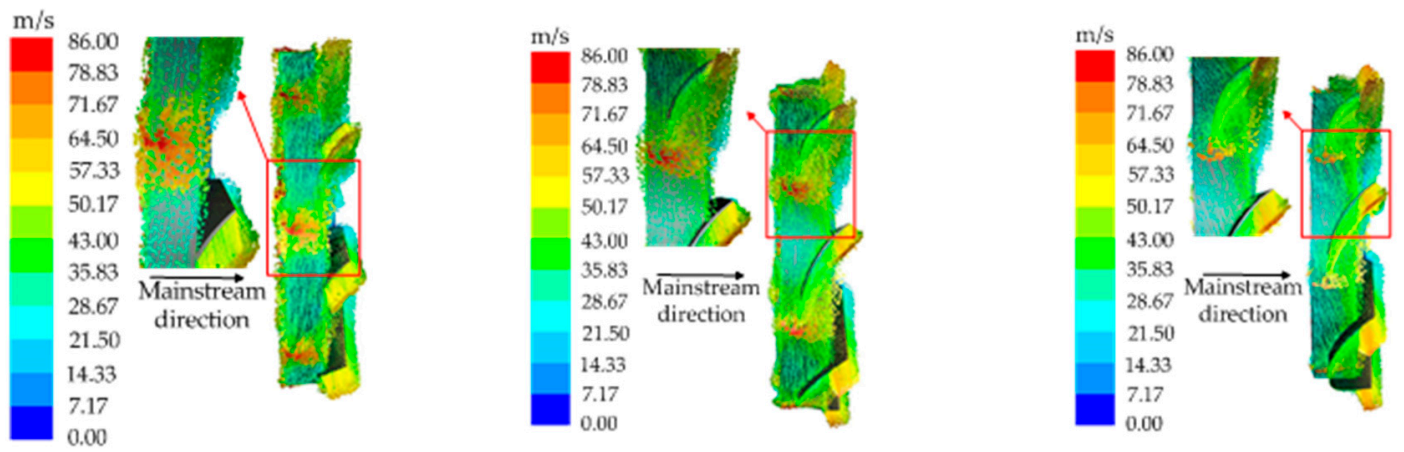

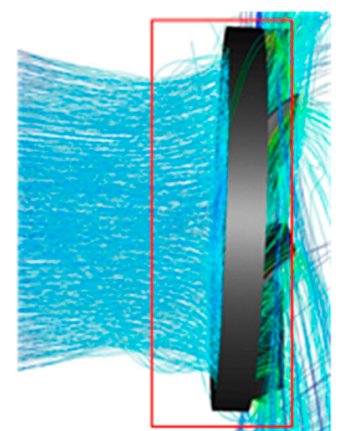

(a)

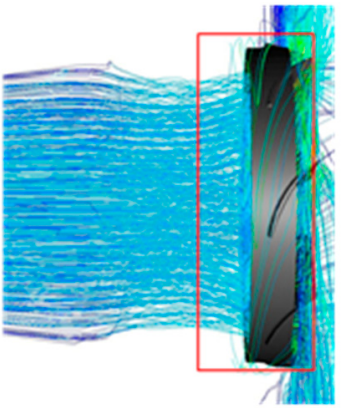

(b)

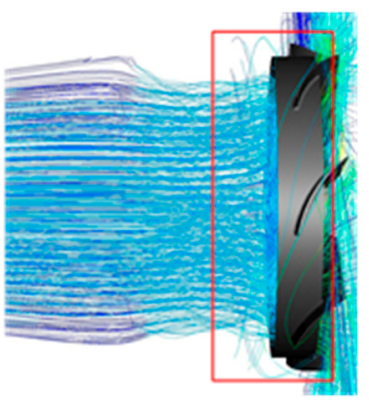

(c)

Figure. 15. Velocity contour profiles comparisons for annular fans with 3 different radial blade extension outer rings : (a) $L r=0 \mathrm{~mm}$, (b) $L r=10 \mathrm{~mm}$, (c) $L r=30 \mathrm{~mm}$ 

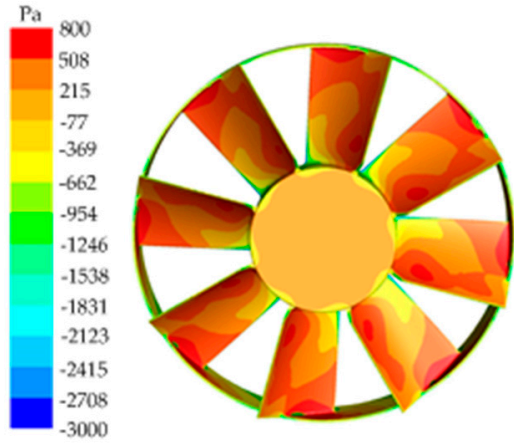

(a)

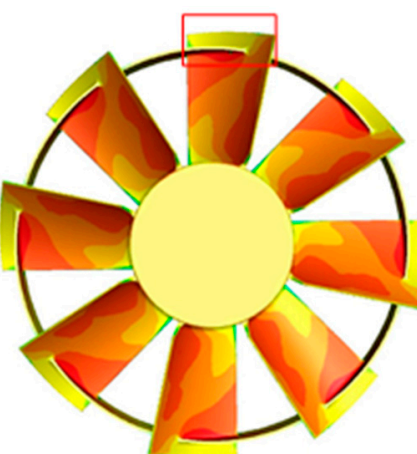

(b)

Figure. 16. Static pressure contour profiles comparisons for annular fan with 2 different radial blade extension

$$
\text { outer rings: (a) } L r=0 \mathrm{~mm} \text {, (b) } L r=30 \mathrm{~mm}
$$

As shown in Figure. 16, the static pressure contour profiles on blades of the 2 fans are similar, but negative pressure region is observed on the blade tip marked in Figure. 16(b). The region mentioned above is produced for the complex blade shape and high rotating speed. However, the pressure value of this region is lower than the inside of the outer ring. Therefore, the answer is that a quantifiable pressure difference is produced here that leads to another back flow around the outer ring as shown in Figure. 15(b) and (c). In conclusion, lower static pressure efficiency is caused by these reasons.

It should be pointed that: during the research process, the cooling fan diameter remains unchanged, and because the blade extension length changes as well as the diameter of the outer ring changes. In addition, another conclusion can be drawn: if the radial blade extension is far smaller than the fan diameter, the mainstream would distribute in the interior zone of the outer ring. Furthermore, if the other fan parameters are kept constant, the annular fan has a worse aerodynamic performance with a smaller outer ring diameter. Based on the similarity law of fan [1], the static pressure and fan power decrease as the ring diameter decreases at the same flow rate. However, the static efficiency of the fan does not change.

\subsection{Effects of the Outer Ring Shapes}

As shown in Figure. 8, shapes of outer ring can be categorized into non-chamfer shape (shape 3) and chamfer shapes (shape 1 and 2). The chamfer shapes are categorized into front-edge-wall (upstream of the airflow) chamfer (shape 2) and rear-edge-wall (downstream of the airflow) chamfer (shape 1). Effects on aerodynamic performance for annular fan with 3 different outer ring shapes are studied in this section.

Figure. 17 shows that the annular fan with shape 1 outer ring has the maximum static pressure and static pressure efficiency. The annular fan with shape 2 outer ring has the minimum static pressure and fan power. The annular fan with shape 3 outer ring has the largest fan power, but has the lowest static pressure efficiency. 


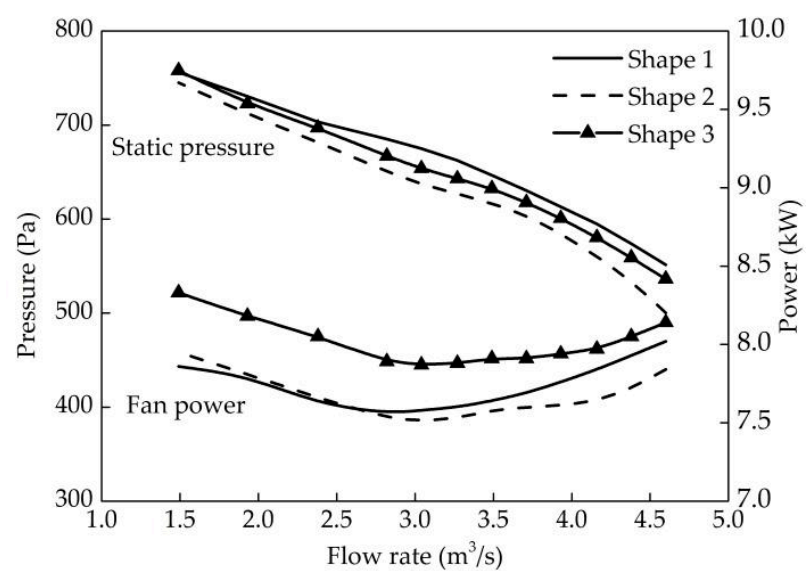

(a)

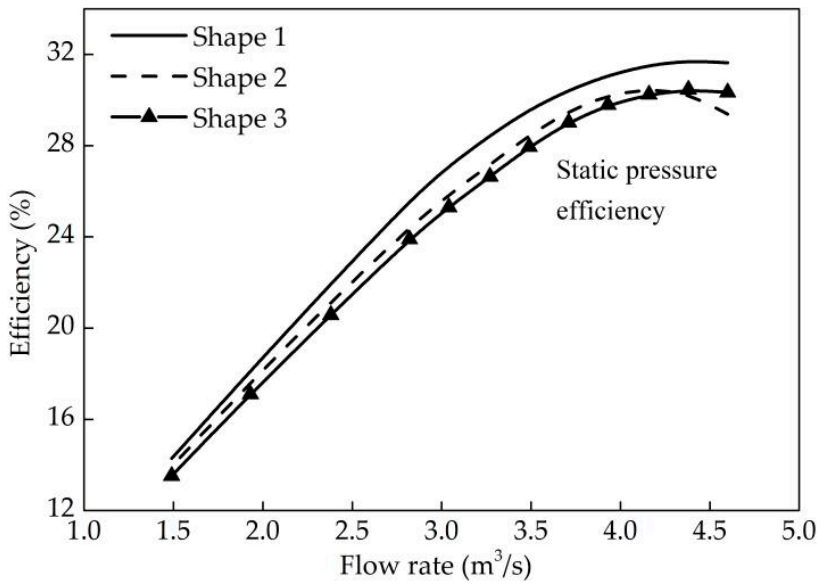

(b)

Figure. 17. Comparisons of aerodynamic performance for annular fan with outer ring with 3 different outer ring shapes:

(a) static pressure \& Fan power, (b) static pressure efficiency

Based on the velocity contour profiles of annular fans with three different shape outer rings (as shown in Figure. 18), backflow is produced in all flow fields within 3 different fans. The area values of backflow are similar to each other. But the velocity values of backflow are markedly different. For annular fan with shape 2 outer ring, the backflow area is smallest. But the velocity value is smallest for annular fan with shape 1 outer ring. And the air would be smoothed furthest when it flows through the front-edge fillet structure, which can reduce the flow loss along the flow path. Therefore, a proper fillet structure can improve the aerodynamic performance for the annular cooling fan.
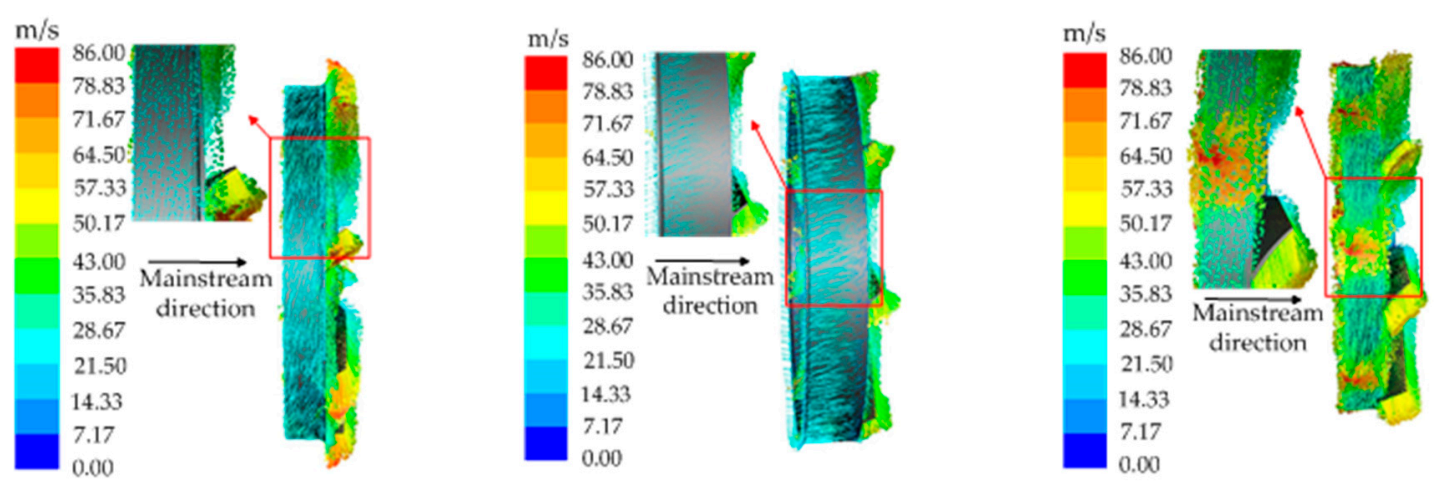

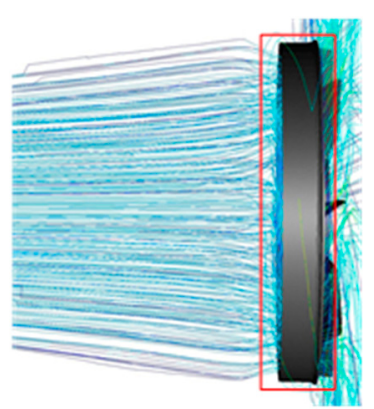

(a)

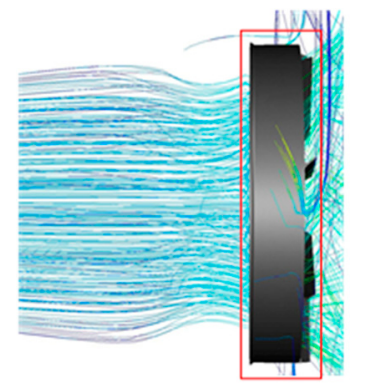

(b)

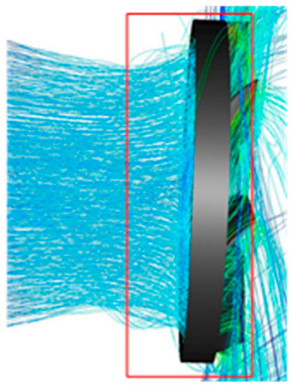

(c)

Figure. 18. Velocity contour profiles comparisons for annular fan with 3 different shape outer rings: (a) shape 1

(b) shape 2, (c) shape 3 


\subsection{Final Design Application}

In this section, the correctness of the design method for the outer ring is validated by test. As shown in Figure. 19, three cooling fans are selected for the test on the aerodynamic performance test bench. The three fans have the same diameter and blade profile as mentioned in previous sections. Fan (a) is a common cooling fan without outer ring. Interim annular cooling fan (b) with outer ring has aperture opening rate of $8.4 \%$, the radial extension length of the blade is $0 \mathrm{~mm}$, and the arc chamfer structure is designed at the downstream side. The final annular fan (c) with outer ring has the same structure parameters as fan (b) but the aperture opening ratio is $46.8 \%$. Three designed physical fans are shown in Figure. 19.

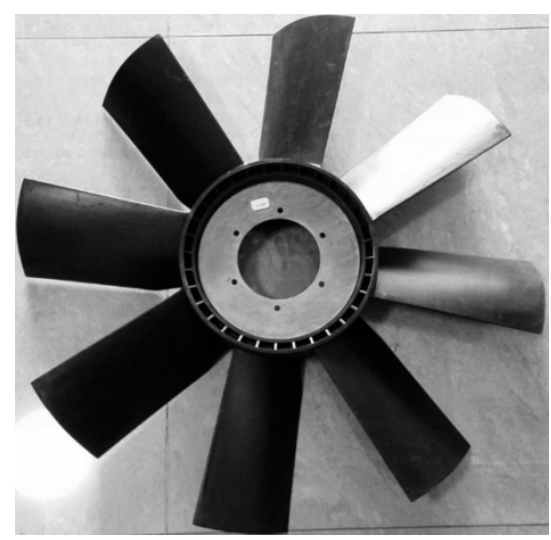

(a)

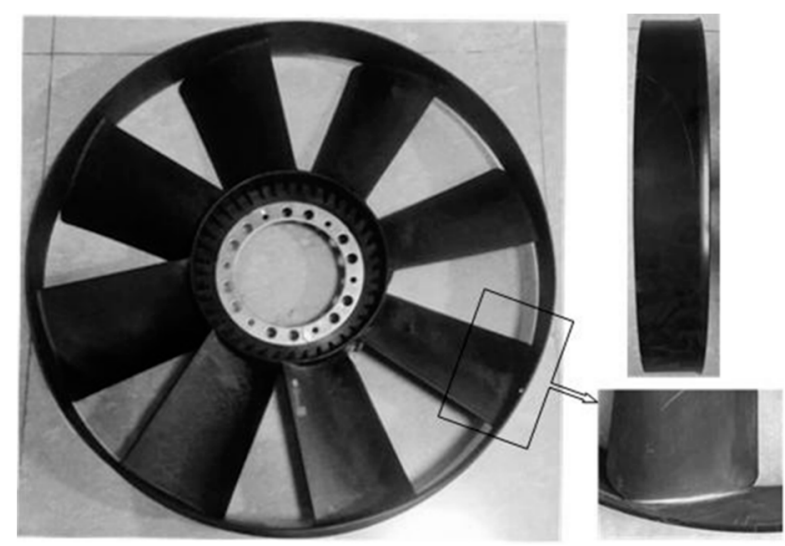

(b)

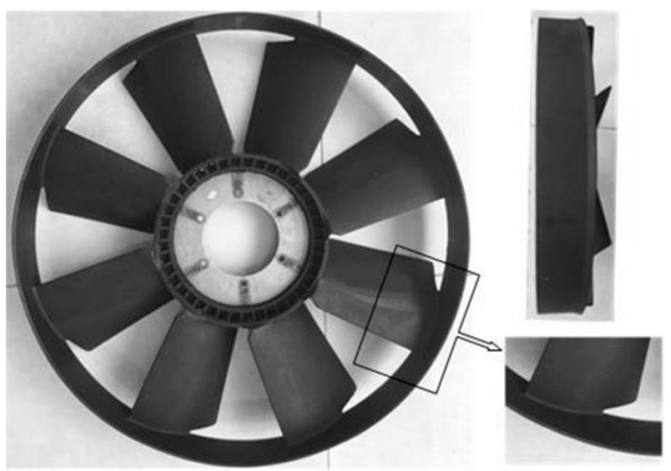

(c)

Figure. 19. Structure comparisons of 3 different cooling fans: (a) common fan without outer ring (b) interim fan with outer ring and $i=8.4 \%, L r=0 \mathrm{~mm}$ (c) final annular cooling fan with outer ring and $\mathrm{i}=46.8 \%, L r=0 \mathrm{~mm}$

All three fans are tested on the test pipeline bench and the test data are obtained to be compared. From Figure. 20, it can be found that both the static pressure and the fan power are significantly increased after adding the outer ring. At the flow rate of $3.8 \mathrm{~m}^{3} / \mathrm{s}$, the static pressure increases with a degrees of $15.4 \%$ and $9.6 \%$ for the final annular fan and the interim fan, respectively. However, the static pressure efficiency for the interim cooling fan is lower than the common fan at all flow rates, and the largest decline of it reaches $6.1 \%$ at the flow rate of $2.9 \mathrm{~m}^{3} / \mathrm{s}$. The main reason of it is that the outer ring has a wider axial projection width and a larger back-flow area would be produced based on the pattern shown in Figure. 13.

In conclusion, based on the evaluation index of static pressure, the aerodynamic performance of annular cooling fan with outer ring is better than common fan without outer ring. However, the aerodynamic performance of the cooling fan cannot be always improved by adding an outer ring. 


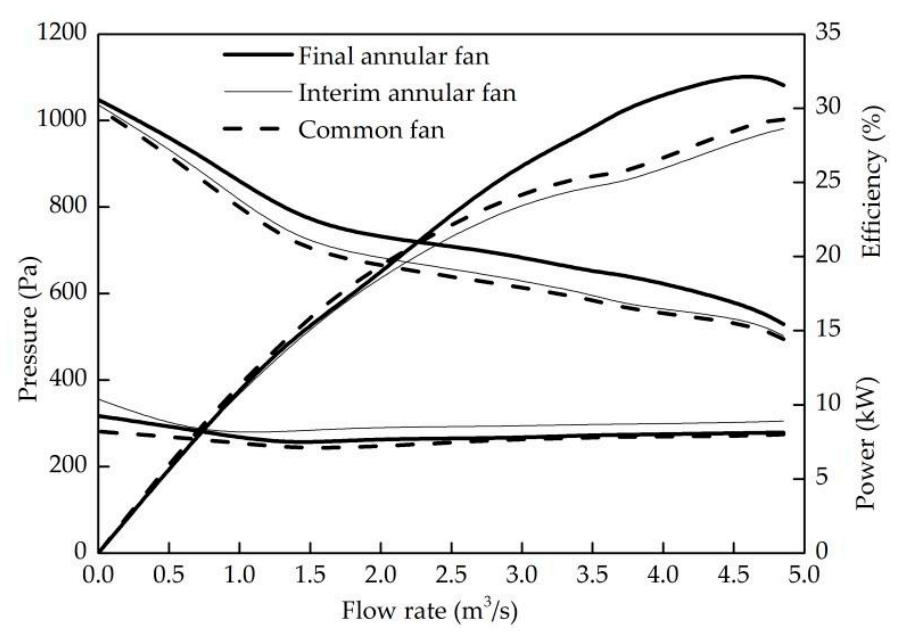

Figure. 20.Aerodynamic performance comparisons for 3 different cooling fans

\section{Conclusions}

In this paper, the aerodynamic performance indexes of engine axial flow cooling fan are described. The test and simulation methods of the cooling fan are introduced. Besides, the accuracy of the simulation model is verified.

Based on the "small winglet" structure, annular cooling fan with outer ring is designed. Three important structural parameters of the outer ring are defined and discussed.

The aperture opening ratio and shape of the outer ring have great influence on the aerodynamic performance of the annular cooling fan, while the length of blade extension has minor influence on the performance. Therefore, when designing the outer ring, the aperture opening ration and shape of the outer ring are the primary parameters to be considered.

If the flow rate remains constant, the aerodynamic performance increase first and then decrease with the increase of aperture opening ratio. Therefore, it is necessary ti select the appropriate parameter value of the structure to optimize the aerodynamic performance.

The downstream side of the outer ring can smoothly guide the air flow. Because the diameter of the outer ring increases gradually along the flow direction, it can accelerate air velocity, reduce the possibility of backflow and improve the flow condition.

\section{References}

1. Bleier, F. P., Fan Handbook: Selection, Application, and Design, 1st ed.; McGraw- Hill: New York, USA , 1997; pp. 33-151.

2. Hennissen, J., Modeling of axial fans for electronic equipment, Eurotherm Seminar 45th ed, Washington D C, USA, 1995; 241-250.

3. Tallman, J., Lakshminarayana, B., Numerical simulation of tip leakage flows in axial flow turbines, with emphasis on flow physics: Part I-Effect of tip clearance height, J. Turbomach, 2000, 123, 314-323.

4. Tallman, J., Lakshminarayana, B., Numerical simulation of tip leakage flows in axial flow turbines, with emphasis on flow physics: Part II-Erect of outer casing relative motion, J. Turbomach, 2000, 123, 314-323.

5. Han, S. B., Zhong, J. J., Effect of blade tip winglet on the performance of a highly loaded transonic compressor rotor, Chinese J. Aeronaut, 2016, 29, 653-661. 
6. Chen, H. S., Tan, C. Q, Numerical and experimental study on effects of hub leakage on performance and flow field of axial fan, J. FMI, 2006, 34, 1-6.

7. Wu,Y., Effect of hub-ratio on performance of asymmetric dual-rotor small axial fan, J. Fluid Dynam, 2013, 3, 81-84.

8. Storer, J. A., Cumpsty, N. A., An approximate analysis and prediction method for tip clearance loss in axial compressors, J. Turbomach, 1994, 116, 648-656.

9. Liu, X., Design and prediction of three dimensional flows in a low speed highly loaded axial flow fan, IJFMS, 2013, 6, 94-104.

10. Li, M., Off-design performance analysis of axial flow fan in helicopter, J. Donghua University (English Ed), 2011, 28, 23-30.

11. Norimasa, S., Ying, Z. J., Flow fields with tip leakage vortex in a small axial cooling fan, J. Therm Sci, 2008, $17,156-162$.

12. Du,J., Lin, F, Numerical investigation on the self-induced unsteadiness in tip leakage flow for a transonic fan rots, J. Turbomach, 2010, 132,1-9.

13. Li, Z., Jin, Y. Z., Effect of tip flange on tip leakage flow of small axial flow fans, J. Therm Sci, 2014, 23, $45-52$.

14. Gong, H. L., Je, H. B., Structure of tip leakage flow in a forward-swept axial-flow fan, Flow Turbul Combust, 2014, 70, 241-265.

15. Shigemitsu, T., Fukuda, H., Unsteady flow condition between front and rear rotor of contra-rotating sized axial fan, J. Fluid Dynam, 2017, 20, 371-385.

16. Zhu, T., Experimental and numerical investigation of the tip clearance noise of an axial fan, ASME Turbo Expo, 2013, 35, 1-13.

17. Sasaki, S., Fukuda,M., Prediction of aerodynamic noise in a ring fan based on wake characteristics, J. Therm Sci, 2011, 20, 144-149.

18. Day, I. J., Active suppression of rotating stall and surge in axial compressors, J. Turbomach, 1993,115, 1-8.

19. Shangguan, W. B, Mo, W. B.., Study on the effect of structure and parameters of engine annular cooling fans on aerodynamic performances, Chinese ICEE, 2017, 38, 56-62.

20. Nashimoto, A., Akuto, T., Aerodynamic noise reduction by use of a cooling fan with winglets, $S A E$ Technical-Paper, doi: org/10.4271/2003-01-0531.

21. Nashimoto, A., Fujisawa, N., Measurements of aerodynamic noise and wake flow field in a cooling fan with winglets, J. Visual, 2004, 7, 85-92.

22. Ota, H., Yuichi, K., Development of high efficient radiator cooling fan for automotive application, $S A E$ Technical-Paper, doi: org/10.4271/2013-01-1293. 
409 23. Zhou, Z. H., Chen, S. W., Experiment study of aerodynamic performance for the suction-side and 410 pressure-side winglet-cavity tips in a turbine blade cascade, Exp Therm Fluid Sci, 2018, 90, 220-230.

411 24. Jiang, Y., Zhang, B., CFD study of a new annular lift fan configuration with high lift efficiency, MDPI $412 \quad$ Aerospace, 2017, 04, 1-13.

413 25. Wu, Y. M., Jin, Y. Z., Effect of hub-ratio on performance of asymmetric dual-rotor small axial fan, J. Fluid 414 Dynam, 2013, 03, 81-84. 